\title{
Hot-footed towards cold fusion
}

The only published account so far of thermonuclear fusion in an electrochemical cell raises, as its authors say, "more questions than it provides answers".

Thermonuclear fusion, which allows stars to shine, must also occur in more humdrum circumstances. Put two deuterons together in a deuterium molecule, for example, and there is a small chance that they will fuse together spontaneously, either to produce a tritium nucleus and a proton or a helium-3 nucleus and a neutron. Perhaps luckily, the chance is astronomically small, maybe $10^{-70}$ per molecule per second. At that rate, there would be roughly one fusion event an hour in a quantity of molecular deuterium roughly as massive as the Galaxy.

The reason why muons have been advocated as catalysts of fusion is that their mass (some 200 times greater than that of an electron) makes for a smaller $\mathrm{D}_{2}$ - ion, thereby increasing the wave function of one deuteron at the position of the other and so increasing the still-random chance of fusion.

The great excitement in the past three weeks about the prospect that thermonuclear fusion has been accomplished electrochemically is an extension of this principle. The article by M. Fleischmann and S. Pons which appeared last week $(J$. Electroanalyt. Chem. 261, 301; 1989) begins from the view that conditions favourable for fusion may be created electrochemically by exploiting the familiar affinity of palladium for hydrogen (in all its isotopic forms).

As described, the experiments seem straightforward. Use a platinium anode, a palladium cathode and an electrolyte which is a heavy-water solution of deuterated lithium hydroxide. To prevent the deuterons bubbling away as deuterium, arrange that there is a substantial negative over-voltage on the cathode. The effect is that deuterons accumulate in the palladium lattice, and will continue to do so until their conversion into molecules resumes.

That point, Fleischman and Pons argue, is determined by the chemical potential of the deuterons in the palladium lattice, which is itself determined by the negative over-potential on the cathode. They use the term "galvanostatic compression" to describe this process of forcing deuterons into the palladium.

Three kinds of measurements are described, one of which is simple calorimetry. The question is whether the heat produced in such a cell is greater than expected, which is most simply that calculated by multiplying together the current and the voltage.

The article describes measurements with cells containing electrodes of different shapes and sizes. For three-rod electrodes of different diameters, the specific rate of excess heat production is reported to have increased with increasing current density and with the thickness of the rod (4 $\mathrm{mm}$ at its maximum), both of which argue for a phenomenon liberating excess heat in which the bulk of the palladium is involved. There is also an account of how, in one experiment, the cathode (a $1 \mathrm{~cm}$ cube of palladium) vaporized.

True fusion should of course be recognizable in other ways, by the detection of its by-product particles for example. The authors report measurements of $\gamma$-rays presumed to have occurred by the reaction of neutrons from fusion reactions with protons in the water-bath surrounding the electrolytic cell and also the detection of neutrons (at roughly three times the intensity of the cosmic-ray background) while one experiment was running. They have also looked for (and claim to have found) tritium in the residual electrolyte in a cell.

So does this not add up to a proof of fusion? From the details published so far, no-one can say. The nuclear evidences offered are all close to the edge of what is measurable. The measurement of tritium production, for example, looks convincing, but there are pitfalls. Any quantity of deuterium-rich water invariably contains the heavier isotope in some quantity. One is looking for a small increase in tritium content, rather than its mere presence where there was none before. Similarly, there are other sources of neutrons and gamma rays besides fusion reactions (notably cosmic rays and natural radioactivity), and these must be convincingly subtracted.

Even taking the $\gamma$-ray and neutron production at face value, far too few of either are recorded to explain the claimed heat generation by known fusion reactions. In one of the experiments described, the rate of production of excess heat suggests that there should be between $10^{\mathrm{i}}$ and $10^{14}$ fusion events a second, but the nuclear physics data suggest that known fusion reactions (leading either to tritium or ${ }^{3} \mathrm{He}$ ) account for only about $10^{4}$ of these.

This is the most serious impediment to belief. The heat production in these electrolytic cells is more than a million times greater than nuclear by-products would suggest it can be. This leads Fleischmann and Pons to say that "the bulk of the energy release is due to a hitherto unknown nuclear process or processes". What, one wonders, can that process be? Is it likely to have escaped the attention of nuclear physicists in the past half century?

Sceptics, in the circumstances, will be quick to ask whether the necessary subtle subtractions from the gross energy output have been accurately made - the electrical energy put in, the heat that would have been produced in a normal electrolytic cell (with ordinary hydrogen instead of deuterium) and so on. Because Fleischmann and Pons spend days, perhaps weeks, loading their electrodes with deuterium, there is plenty of energy stored up in their system before any return is obtained. Even the much-described "explosion" of one of their electrodes may be explicable by chemistry.

By now, the tiny band of Nature referees which has scrutinized the two articles submitted for publication has been joined by many other people. Further information about the issues which have given them difficulty will be given in a further issue of Nature. Numerous attempts to replicate the measurements have produced only one positive claim (see page 529). One common complaint has been that the data provided are insufficient to allow faithful reproduction of the measurements reported. The Fleischman and Pons article as now published is a starting-point for other experiments, but says less than the average chemist needs to know.

A more perplexing circumstance is that the authors are reported to have said that some cells work and others do not, although that may not be surprising when so little is known of the system as a whole. The idea that cells produce excess heat only after they have been running for long periods makes sense if a palladium lattice must be charged with deuterons before fusion occurs at anything like a decent rate, but one is left wondering whether the amount of charging needed can be predicted in advance for a given cell geometry and voltage, as one would expect if such a straightforward explanation were the only one. What seems plain is that arguments like these will continue for a long time. 